\title{
PD01 - Respiratory allergens in human milk: potential impact on susceptibility to allergic airway disease
}

\author{
Patricia Macchiaverni ${ }^{{ }^{*}+}$, Akila Rekima ${ }^{2+}$, Mathilde Turfkruyer ${ }^{2}$, Laurent Mascarell ${ }^{3}$, Sabi Airouche ${ }^{3}$, \\ Philippe Moingeon ${ }^{3}$, Karine Adel-Patient ${ }^{4}$, Antonio Condino-Neto ${ }^{1}$, Isabella Annesi-Maesano ${ }^{5,6}$, Susan L Prescott ${ }^{7,8}$, \\ Meri K Tulic ${ }^{2,7,8}$, Valérie Verhasselt ${ }^{2,8}$
}

From 3rd Pediatric Allergy and Asthma Meeting (PAAM)

Athens, Greece. 17-19 October 2013

\section{Background}

Impact of exposure to environmental allergens during early life on allergic sensitization and disease development is controversial.

\section{Objective}

We investigated whether airborne allergen from Dermatophagoides pteronyssinus (Der p), a major cause of allergic asthma, is found in human breast-milk and examined its impact on allergic outcome in a mouse model.

\section{Methods}

Der p 1 was quantified in human colostrum and milk samples from Brasil, Australia and France by ELISA. Basophil degranulation assay was used to confirm immunogenicity of Der p. BALB/c mice were fostered by mothers exposed to Der p during lactation. Progeny allergic response to Der p was measured at 6-weeks.

\section{Results}

Der p 1 was present in 58\% Brazilian, 70\% French, and $78 \%$ Australian colostrum. Median [Der p 1] was similar between countries $(96 \mathrm{pg} / \mathrm{mL})$. In mature milk, Der p1 was found in $55 \%$ of samples, median [Der p 1] was $65.9 \mathrm{pg} / \mathrm{mL}$ and was significantly lower than in colostrum $(\mathrm{p}=0.0001)$. Der $\mathrm{p}$ 1-containing milks were able to induce basophils degranulation. Mice breastfed by Der p-exposed mothers had 5-fold increased levels of Der p specific IgE and IgG1 compared to mice breastfed by naïve mothers. Their allergic airway inflammation was not affected.

\section{Conclusion}

Early life exposure to ubiquitous respiratory allergens can take place through breastfeeding. An animal model mimicking the human situation shows early life exposure to Der $\mathrm{p}$ through milk primes the immune system. The presence of respiratory allergens in breast-milk may be an important factor in driving the early immune function towards allergic disease.

\section{Authors' details \\ 'Institute of Biomedical Sciences, University of São Paulo, São Paulo, Brazil. 2EA 6302 "Tolérance Immunitaire", Université de Nice Sophia-Antipolis, Hôpital de I'Archet, Nice, France. ${ }^{3}$ Research and Development, Stallergenes SA, Antony, France. ${ }^{4}$ INRA, UR496 Immuno-Allergie Alimentaire, CEA/IBiTeC-S/ SPI, CEA de Saclay, F-91191 Gif sur Yvette cedex, France. ${ }^{5}$ EPAR UMR-S 707 INSERM, France. ${ }^{6}$ EPAR UMR-S 707 UPMC Paris6, Medical School Saint- Antoine, Paris, France. ${ }^{7}$ School of Pediatrics and Child Health, University of Western Australia, Perth, Australia. ${ }^{8}$ The International Inflammation "in- FLAME" Network, Worldwide Universities Network (WUN).}

Published: 28 February 2014

doi:10.1186/2045-7022-4-S1-P1

Cite this article as: Macchiaverni et al:: PD01 - Respiratory allergens in human milk: potential impact on susceptibility to allergic airway disease. Clinical and Translational Allergy 2014 4(Suppl 1):P1. 